\title{
Immunocomprised Heart Transplant Patient With Cryptosporidial Diarrhea
}

\author{
Michael Dominic Lee, MSIII, Rajesh Kabadi, MD, Siva K. Kumar, MD
}

\section{Case Presentation}

A 22 year-old female with a past medical history of postpartum cardiomyopathy status post orthotopic heart transplant (OHT) was admitted for diarrhea of 12 days' duration. The diarrhea was watery, non-bloody, and occurring at a rate of 10 episodes per day. She denied nausea, vomiting, fever, or chills, but reported abdominal cramps. On physical exam, she was afebrile, and vital signs were stable. The patient had a regular heart rate and rhythm. Lung sounds were clear to auscultation bilaterally. Her abdomen was soft, non-tender, without rigidity, guarding, or distention. There was no edema in the extremities.

Laboratory studies were all within normal limits. The patient's CMV quantitative analysis, adenovirus culture, CMV culture, blood cultures, fecal culture and C. difficile EIA tests were negative. In addition, serum antigens for Rotavirus, Entameoba Histolytica, Giardia, and Cryptosporidium were negative. Finally, a flexible sigmoidoscopy was performed to define the etiology of her diarrhea and demonstrated erythematous mucosa in the sigmoid. Biopsies were taken in the sigmoid mucosa and the colon. Five days later, the biopsies in both the sigmoid mucosa and colon revealed the presence of scattered organisms suspicious for cryptosporidium.

Before the results of the biopsies were back, the decision was made to discontinue the patient's mycophenolate mofetil $\left(\right.$ Cellcept $\left.^{\circledast}\right)$. Mycophenolate mofetil is normally part of the immunosuppressant regimen used to prevent rejection in organ transplantation. One of the common adverse effects of mycophenolate mofetil is diarrhea. Holding mycophenolate mofetil had the added benefit of lessening its suppressive effects on the patient's immune system. After the discontinuation of

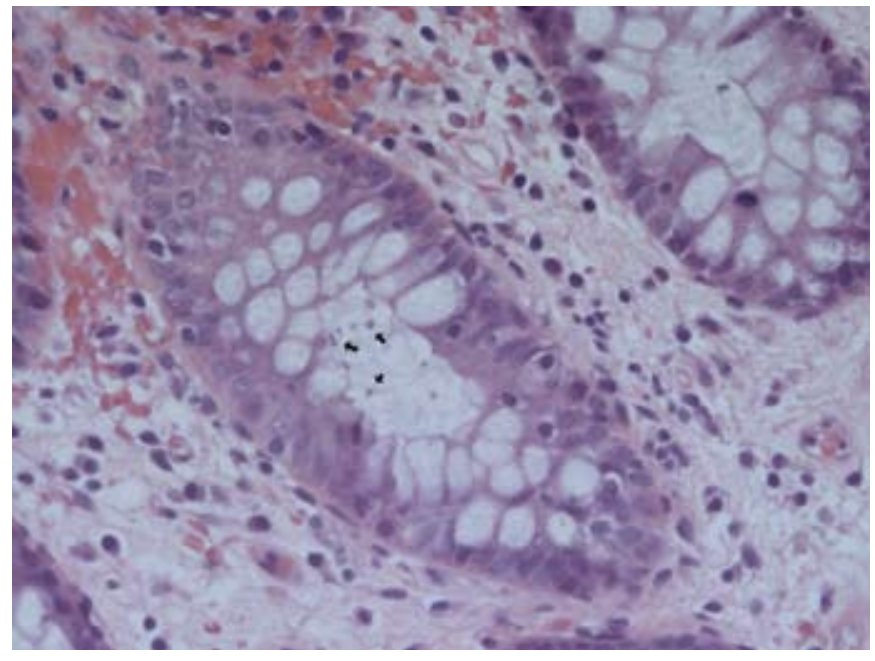

Figure 1. Arrows point to Cryptosporidial organisms located intracellularly within bowel tissue. the mycophenolate mofetil, the patient's diarrhea finally began to abate to less than two episodes of semi-watery diarrhea per day. The patient was able to tolerate an oral diet and no longer had abdominal cramps. The patient was discharged a day later with instructions to discontinue mycophenolate mofetil.

\section{Discussion}

Cryptosporidium is an intracellular protozoan parasite that is recognized as a major cause of diarrhea in immunosuppressed hosts. This includes patients with HIV, organ transplantation, IgA deficiency, hypogammaglobulinemia, and on immunosuppressive therapy. In the United States and Europe, 8-30\% of HIV patients excrete Cryptosporidium oocysts, and in developing countries, this figure climbs to $15-50 \%$, making it one of the most common enteropathogens. ${ }^{1}$ The prevalence in HIVnegative, immunocompromised patients is unclear.

Diagnosis of Cryptosporidiosis can be tricky because there are no characteristic laboratory findings indicative of Cryptosporidium infection other than identification of the organisms. As a result, the diagnosis is primarily based on microscopic identification of the oocysts in stool or tissue. Other sites to look for the organisms include duodenal aspirates, bile secretions, biopsy specimens from affected gastrointestinal tissue, or respiratory secretions. Diagnosis is also complicated by the low sensitivity of ova and parasite tests in stool. In one report, examination of a single stool specimen identified only $30 \%$ of intestinal Cryptosporidial infections. ${ }^{2}$ Therefore, it is recommended to test three stool specimens before excluding the diagnosis of Cryptosporidiosis.

Finally, there is currently no reliable therapy for Cryptosporidiosis. Recovery from Cryptosporidiosis is based upon the immune status of the patient. Immunologically competent patients usually have a spontaneous recovery within a few weeks without requiring any specific therapy. For immunocompetent children, nitazoxanide is the drug of choice. For HIV-infected patients, the recommendation is to initiate HAART in order to restore immune system function. Restoration to a CD4 count $>100$ cells/microL is associated with complete resolution of symptoms. ${ }^{3}$ There are currently no recommendations regarding how to treat HIV-negative, immunocompromised patients.

\section{References}

1. Jelinek, T, Lotze, M, Eichenlaub, S, et al. Prevalence of infection with Cryptosporidium parvum and Cyclospora cayetanensis among international travellers. Gut 1997; 41:801.

2. Blanshard, C, Jackson, AM, Shanson, DC, et al. Cryptosporidiosis in HIVseropositive patients. Q J Med 1992; 85:813.

3. Abubakar, I, Aliyu, Sh, Arumugam, C, et al. Prevention and treatment of cryptosporidiosis in immunocompromised patients. Cochrane Database Syst Rev 2007; CD004932. 